\title{
Hippocampal GFAP-positive astrocyte responses to amyloid and tau pathologies
}

Marco Antônio De Bastiani, $\mathrm{PhD}^{1^{*}}$, Bruna Bellaver, $\mathrm{PhD}^{1,2^{*}}$, Wagner S. Brum ${ }^{1,3}$, Debora G. Souza, $\mathrm{PhD}^{1}$, Pamela C. L. Ferreira, $\mathrm{PhD}^{2}$, Andreia S. Rocha, $\mathrm{MA}^{1}$, Guilherme Povala, $\mathrm{MA}^{1}$, João Pedro Ferrari-Souza ${ }^{1,2}$, Andrea L. Benedet, $\mathrm{PhD}^{3}$, Nicholas J. Ashton, $\mathrm{PhD}^{3,4,5,6}$, Thomas K. Karikari, PhD ${ }^{2,3,4}$, Henrik Zetterberg, MD. $\mathrm{PhD}^{3,4,7,8,9}$, Kaj Blennow, MD. PhD ${ }^{3,4}$, Pedro Rosa-Neto MD. $\mathrm{PhD}^{10}$, Tharick A. Pascoal, MD. $\mathrm{PhD}^{2}$, Eduardo R. Zimmer, $\mathrm{PhD}^{1,11,12}$, for the Alzheimer's Disease Neuroimaging Initiative

${ }^{1}$ Graduate Program in Biological Sciences: Biochemistry, Universidade Federal do Rio Grande do Sul, Porto Alegre, RS, Brazil.

${ }^{2}$ Department of Psychiatry, University of Pittsburgh, Pittsburgh, PA, USA.

${ }^{3}$ Department of Psychiatry and Neurochemistry, The Sahlgrenska Academy at the University of Gothenburg, Mölndal, Sweden.

${ }^{4}$ Clinical Neurochemistry Laboratory, Sahlgrenska University Hospital, Gothenburg, Sweden.

${ }^{5}$ Wallenberg Centre for Molecular and Translational Medicine, University of Gothenburg, Gothenburg, Sweden.

${ }^{6}$ Department of Old Age Psychiatry, Institute of Psychiatry, Psychology \& Neuroscience, King’s College London, London, UK.

${ }^{7}$ Department of Neurodegenerative Disease, UCL Queen Square Institute of Neurology, London, UK.

${ }^{8}$ UK Dementia Research Institute at UCL, London, UK.

${ }^{9}$ Hong Kong Center for Neurodegenerative Diseases, Hong Kong, China.

${ }^{10}$ Translational Neuroimaging Laboratory (TNL), McGill Center for Studies in Aging (MCSA), Douglas Mental Health University Institute, Departments of Neurology and Neurosurgery, Psychiatry, and Pharmacology, McGill University, Montreal, Canada.

${ }^{11}$ Department of Pharmacology, Universidade Federal do Rio Grande do Sul, Porto Alegre, RS, Brazil.

${ }^{12}$ Graduate Program in Biological Sciences: Pharmacology and Therapeutics, Universidade Federal do Rio Grande do Sul, Porto Alegre, RS, Brazil.

*Both authors contributed equally to this article

\section{Corresponding author:}

Eduardo R. Zimmer, PhD (E.R. Zimmer)

Department of Pharmacology, Universidade Federal do Rio Grande do Sul (UFRGS)

2500 Ramiro Barcelos street, 90035-003, Porto Alegre, RS, Brazil

Email address: eduardo.zimmer@ufrgs.br

Telephone: +55 5133085558

Fax: +55 5133085544

Website: www.zimmer-lab.org 


\begin{abstract}
Introduction: In Alzheimer's disease clinical research, glial fibrillary acidic protein (GFAP) released in the cerebrospinal fluid and blood is widely measured and perceived as a biomarker of reactive astrogliosis. However, it was recently demonstrated that plasma GFAP levels are associated with amyloid- $\beta$ (A $\beta)$ but not tau pathology. The molecular underpinnings behind this specificity are unexplored. Here we investigated biomarker and transcriptomic associations of GFAP-positive astrocytes with $\mathrm{A} \beta$ and tau pathologies in humans and mouse models.

Methods: We studied 90 individuals with plasma GFAP, A $\beta$ - and Tau-PET to investigate the association between biomarkers. Then, transcriptomic analysis in hippocampal GFAPpositive astrocytes isolated from mouse models presenting A $\beta$ (PS2APP) or tau (P301S) pathologies was applied to explore differentially expressed genes (DEGs), Gene Ontology processes, and protein-protein interaction networks associated with each phenotype.
\end{abstract}

Findings: In humans, we found that plasma GFAP associates with $A \beta$ but not tau pathology. Supporting these results, mouse transcriptomics showed scarce overlap of DEGs between the $\mathrm{A} \beta$ and tau mouse models, revealing the unique nature of GFAP-positive astrocytic responses to $A \beta$ or tau pathology. While $A \beta$ GFAP-positive astrocytes were overrepresented with genes associated with proteostasis and exocytosis-related processes, tau hippocampal GFAPpositive astrocytes presented greater abnormalities in functions related to DNA/RNA processing and cytoskeleton dynamics.

Interpretation: Our results offer insights into $A \beta$ - and tau-driven specific signatures in GFAP-positive astrocytes. Characterizing how different underlying pathologies distinctly influence astrocyte responses is critical for the biological interpretation of astrocyte-related biomarker and suggests the need to develop context-specific astrocyte targets to study AD.

Funding: This study was supported by Instituto Serrapilheira, Alzheimer's Association, CAPES, CNPq and FAPERGS.

Keywords: biomarker, GFAP, amyloidosis, tau pathology, Alzheimer’s disease. 


\section{Evidence before the study}

The glial fibrillary acidic protein (GFAP) is commonly used as a proxy of reactive astrogliosis in Alzheimer's disease (AD) clinical studies. Recent evidence demonstrated that increases in GFAP levels are associated with a specific aspect of AD pathology, namely amyloid- $\beta(A \beta)$. However, the biological interpretation behind it was not explored in depth molecularly. Uncovering the phenotypes acquired by GFAP-positive astrocytes under A $\beta$ or tau influence might directly impact clinical research by improving the biological interpretation of clinical glial biomarkers used in $\mathrm{AD}$.

\section{Added value of this study}

This study revealed molecular signatures driven by AD hallmarks in hippocampal GFAPpositive astrocytes, including 1) a scarce overlap of differentially expressed genes (DEG) driven by $A \beta$ and tau; 2) abnormalities in proteostasis and exocytosis-related processes in A $\beta$-driven reactive astrocytes and 3) impaired DNA/RNA processing and cytoskeleton dynamics in tau-driven astrocytes. Additionally, this study revealed several astrocyte-related DEGs and biological pathways specific to each aspect of AD core pathology.

\section{Implications of all the available evidence}

These results shed light on the existence of GFAP-positive astrocyte signatures driven by A $\beta$ and tau pathologies in AD. Characterizing how upstream triggers influence GFAP-positive astrocyte molecular signals may contribute to the biological interpretation of GFAP as a biomarker of $\mathrm{AD}$. Additionally, the signature genes and related biological processes identified in this study might guide other studies to identify context-specific astrocyte biomarkers for AD. Finally, it paves the way to unraveling novel mechanisms and therapeutic targets capable of potentiating the effects of emerging anti- $\mathrm{A}_{\text {Lx }}$ and anti-tau therapies. 


\section{Introduction}

Astrocytes are the main homeostatic cells of the brain, which are involved in most aspects of brain physiology. For a long time, the response of astrocytes to brain injury - a process collectively called astrocyte reactivity - was considered an almost universal phenomenon. Nowadays, there is a consensus that glial responses are rather complex, heterogeneous, and context-specific (1-3). In this sense, astrocytes are likely decisive contributors to Alzheimer's disease (AD) onset and progression; however, the molecular underpinnings are still poorly understood. Furthermore, transcriptomics analysis in postmortem $\mathrm{AD}$ brains demonstrated highly variable intra-cohort astrocyte molecular signatures in individuals with the same clinical diagnosis, indicating that specific aspects of $\mathrm{AD}$ pathophysiology might be triggering different astrocyte phenotypes (4). In this context, Jiwaji and colleagues started uncovering the heterogeneity of astrocyte reactivity facing A $\beta$ and tau pathologies, the neuropathological hallmarks of AD (5).

Astrocyte biomarkers are reported to be consistently increased in cerebrospinal fluid (CSF) and blood of AD patients compared to cognitively unimpaired (CU) individuals $(6,7)$. Although it is known that astrocyte reactivity cannot be measured by one single marker, clinical research commonly relies on the measurement of glial fibrillary acidic protein (GFAP) concentration in CSF or blood as a proxy of astrocyte reactivity. It was recently demonstrated that plasma GFAP levels are more associated with the A $\beta$ burden than tau pathology in $\operatorname{AD}(8,9)$ - an association that was found to be more robust in blood than in CSF. In this sense, uncovering the phenotypes acquired by GFAP-positive astrocytes under $\mathrm{A} \beta$ or tau influence might directly impact clinical research by improving the biological interpretation of clinical biomarkers used in AD.

In this study, we investigated GFAP associations with $A \beta$ and tau pathologies in individuals across aging and AD spectrum. Furthermore, we investigated the molecular signatures of GFAP-positive astrocytes driven by $\mathrm{A} \beta$ or tau, comparing the hippocampal transcriptomic profile of astrocytes from animal models of amyloidosis (PS2APP mice) and tau pathology (P301S mice). Differentially expressed genes (DEGs), protein-protein networks, and functional enrichment analysis of Gene Ontology (GO) terms were performed to identify biological differences and similarities between the phenotypes acquired by these astrocytes. The molecular characterization of astrocyte responses to $A \beta$ and tau pathology may pave the way for a better understanding of the GFAP biological interpretation in AD.

\section{Methods}




\section{Human Cohort}

Data used in the preparation of this article were obtained from the Alzheimer's Disease Neuroimaging Initiative (ADNI) database, phases GO and 2. ADNI was launched in 2003 as a public-private partnership, led by Principal Investigator Michael W. Weiner, MD. The primary goal of ADNI has been to test whether serial magnetic resonance imaging (MRI), PET, other biological markers, and clinical and neuropsychological assessments can be combined to measure the progression of mild cognitive impairment (MCI) and early AD. ADNI study was conducted according to Good Clinical Practice guidelines, US 21CFR Part 50 - Protection of Human Subjects, and Part 56 - Institutional Review Boards, and pursuant to state and federal HIPAA regulations and was approved by the Institutional Review Board of each participating site (adni.loni.usc.edu). Written informed consent for the study was obtained from all participants and/or authorized representatives

Based on biomarker availability, 90 participants were included [CU: $n=39$; cognitively impaired (CI; mild cognitive impairment $(\mathrm{MCI})$ or AD dementia): $n=51$ ] from the ADNI database. All participants had available measurements of plasma GFAP and positron emission tomography (PET) data for $\left[{ }^{18} \mathrm{~F}\right]$-florbetapir (A $\beta$-PET) and $\left[{ }^{18} \mathrm{~F}\right]$ flortaucipir (Tau-PET), within a maximum 2.5-year time interval between scan and plasma collection. Plasma GFAP was measured with Simoa Neurology 4-Plex (\#103670, Quanterix). For A $\beta$-PET data analysis, as recommended for cross-sectional analyses (10), the global cortical composite standardized uptake value ratio (SUVr) normalized by the whole cerebellum was used. When applicable, global A $\beta$-PET cortical composite was used to dichotomize participants into $A \beta$-negative $(A-)$ and $A \beta$-positive $(A+)$ based on the widely validated ADNI cutoff of $>1.11$ (10). For tau-PET, we assessed the temporal meta ROI values and the cutoff for tau-PET positivity of 1.23 was used (11). Baseline characteristics were summarized with descriptive statistics. Pearson correlation was applied to assess associations between biomarkers, and statistical significance was set to $\alpha=0.05$ (FDRadjusted).

\section{RNA-seq Data Acquisition and Differential Expression Analysis}

Expression profiling by high throughput sequencing studies of mice hippocampus GFAP-positive astrocytes were obtained from the NCBI Gene Expression Omnibus (GEO) (https://www.ncbi.nlm.nih.gov/geo). The first study (GSE129770) contains data from 5 PS2APP and 5 wild-type (WT) mice (11.5-months old); the second study (GSE129797) contains data from 5 Tau P301S and 5 WT mice (6-months old) (12). First, raw RNA-seq 
data from both studies was downloaded using the SRA Toolkit (https://github.com/ncbi/sra-

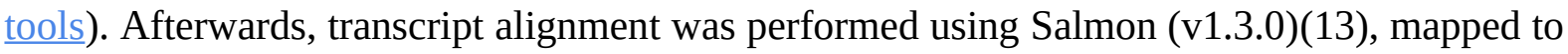
a reference genome with the index derived from Mus musculus GRCm38 Ensembl build. Aligned reads were summarized using tximport (v1.12.3)(14) and genes with mean count < 10 were filtered out. Finally, processed expression data from both hippocampus astrocyte studies was analyzed with the DESeq2 (v1.28.1)(15) method for differential expression evaluation of either PS2APP or Tau P301S mutations versus respective WT mice. Genes with FDR adjusted p-value $<0.1$ were considered as DEGs for further analyses. Venn diagrams were constructed using the VennDiagram package (v1.6.20).

\section{Functional Enrichment Analysis and Network}

Gene Ontology (GO) enrichment analysis of DEGs previously obtained for both studies was implemented using the clusterProfiler (v3.16.1) package (16). The significantly enriched terms from biological process (BP), cellular component (CC) and molecular function (MF) sub-ontologies were used to build networks employing the RedeR (v1.36.0) and GOplot (v1.0.2) packages $(17,18)$. In this network, GO terms are mapped to nodes and edges represent the proportion of gene overlap (Jaccard Coefficient $>0.15$ ) between all pairs of terms.

\section{Protein-Protein Interaction Networks and Circos Plots}

We used the Search Tool for the Retrieval of Interacting Genes/Proteins (STRING) Consortium to build protein-protein interaction (PPI) networks with the DEGs for both datasets. STRING is a biological database and web resource of known and predicted proteinprotein interactions which contains information from numerous sources, including experimental data, computational prediction methods and public text collections. The construction of the networks was implemented in R using the STRINGdb (v2.0.2), RedeR (v1.36.0) and igraph (v1.2.6) packages $(17,19)$. Final network was filtered to retain only gene connections with STRING combined confidence interaction scores $>0.7$. For each PPI network, genes observed in GO terms significantly enriched were manually curated and categorized. These functional categorizations were used to create circos plots showing category linkages and link proportions in PS2APP and Tau P301S networks. The plots were built using the circlize package (v0.4.13)(20).

\section{Results}




\section{Plasma GFAP differently associates with biomarkers of AD pathology}

Table 1 shows demographic information of the 90 individuals (39 CU and $51 \mathrm{CI}$ ) assessed. Correlations between GFAP and other known biomarkers of AD pathology and neurodegeneration showed that GFAP negatively correlated with CSF A $\beta 42 / 40$, but no significant associations were observed with p-Tau181 (Supplemental Fig. 1). We then further investigated associations between $A \beta$-and Tau-PET with plasma GFAP levels in individuals across aging and the $\mathrm{AD}$ continuum. Linear regressions using composite brain regions revealed that both $\mathrm{A} \beta$ - and Tau-PET significantly associated with plasma GFAP (Fig. 1A, C). However, when individuals were stratified by their $A \beta$ status ( $A$ - and $A+$ ), we observed that the association between $A \beta$ - and Tau-PET with plasma GFAP remains significant only in A+ individuals (Fig. 1B, D).

Furthermore, we evaluated whether GFAP levels are associated with changes in TauPET, to further assess how this effect would be affected by including A $\beta$-PET as a covariate. We built models including either (i) Braak III-IV or (ii) Braak V-VI SUVr as the outcomes, with two predictor schemes, plasma GFAP alone or plasma GFAP and A $\beta$-PET global SUVr, resulting in four models. For Braak III-IV SUVr as the outcome, increases in plasma GFAP were associated with increases in Tau-PET ( $\beta$-estimate: 0.054; $\mathrm{t}=3.68$; $\mathrm{p}<0.001$ ). When including plasma GFAP and A $\beta$-PET as predictors, plasma GFAP was no longer associated with increases in Tau-PET ( $\beta$-estimate: 0.019; $\mathrm{t}=1.35 ; \mathrm{p}=0.18$ ). For Braak V-VI as the outcome, the same was observed. In the univariate model, increases in plasma GFAP were associated with increases in Tau-PET ( $\beta$-estimate: 0.031; $\mathrm{t}=2.43 ; \mathrm{p}=0.02$ ). When A $\beta$-PET SUVr was also added as predictor, plasma GFAP was no longer associated with increases in Tau-PET ( $\beta$-estimate: 0.0059; $\mathrm{t}=0.46 ; \mathrm{p}=0.65$ ). Our results corroborate previous studies in other cohorts $(8,9)$, evidencing the specificity of plasma GFAP association with $A \beta$, but not tau pathology.

\section{GFAP-positive astrocytes affected either by $A \beta$ or tau pathology present a scarce overlap of DEGs}

To better understand the biological underpinnings of GFAP association with $A \beta$ pathology, we compared GFAP-positive astrocytes isolated from $A \beta$ and tau mouse models. The amyloidosis model (PS2APP) presented 95 downregulated genes (adjusted p-value < 0.1) compared to WT mice (Fig. 2A). Log fold change values ranged from -2.9365 to 0.4204. The most significantly downregulated gene was Aquaporin 11 (Aqp11), which encodes a water channel protein that also facilitates the transport of glycerol and hydrogen 
peroxide across membranes. In addition, 200 upregulated genes were also found compared to WT mice (adjusted p-value < 0.1) comprising log fold change values ranging from 0.4308 to 4.9284. The most significant upregulated gene was PTPN6, which encodes the Protein Tyrosine Phosphatase Non-receptor Type 6, also known as Src homology region 2 domaincontaining phosphatase-1 (SHP-1), a tyrosine phosphatase enzyme. The Tau model (P301S) presented 178 statistically significant downregulated genes compared to WT mice (Fig. 2B). The expression of Insulin-1 (Ins1) and Insulin-2 (Ins2) were mostly impacted. These genes encode the hormone insulin, and may play a role in growth, differentiation and glucose metabolism in the brain. The other downregulated genes ranged between -4.098 to - $0.507 \log$ fold change and are related to different pathways. In parallel, 427 statistically significant upregulated genes were found in Tau P301S compared to WT mice. The log fold change values ranged from 0.5216 to 4.212 . The most significant upregulated gene identified was Retrotransposon Gag Like 8B (Rtl8b), also known as Mart, considered a neofunctionalized retrotransposon gene. Interestingly, only 30 DEGs overlapped among astrocytes from these two mouse models, revealing $A \beta$ and tau drive specific astrocyte molecular remodeling (Fig. 2C). Results of differential expression analysis for both animal models are presented in Supplemental Table 1.

\section{$A \beta$ and tau promote the enrichment of distinct functional categories in GFAP-positive astrocytes}

The PPI analysis showed a network containing 70 altered nodes in the PS2APP hippocampal astrocytes dataset. The average node degree was 3.285 and the number of edges was 115 (expected: 74). The average local clustering coefficient was 0.521, while the global clustering coefficient was 0.21. Based on the connectivity degree, Uba52, Ubc, Ccnh, Arrb2, and Gng12 were the top 5 hub proteins. The results were plotted as a PPI network (Fig. 3A). Circos plot (Fig. 3B) indicates the altered protein interconnectivity in the network based on their functional categories, with "Proteostasis" being the leading functional alteration in PS2APP astrocytes.

The PPI data analysis showed a different profile of the gene network in the Tau P301S model compared to the PS2APP. PPI analysis of the Tau P301S hippocampal astrocytes showed 204 altered nodes. The average node degree was 4.137 and the number of edges was 422 (expected: 373). The average local clustering coefficient was 0.551, and the global clustering coefficient was 0.5. Based on the connectivity degree, Stat1, Usp18, Irf7, Pik3ca, and Cxcl10 were among the top 5 hub proteins. The results were plotted as a PPI 
network and protein nodes were colored according to their functional category (Fig. 3C). Circos plot (Fig. 3D) indicates the interconnectivity of the altered proteins based on their functional categories, being "DNA/RNA processing", “Inflammation” and "Cytoskeleton” the leading functional alterations. The modulation of gene expression for both networks can be visualized in Supplemental Fig. 2.

\section{$A \beta$ and tau pathologies drive GFAP-positive astrocytes changes in Gene Ontology biological domains}

The functional enrichment analysis revealed 36 GO terms enriched with DEGs altered in the PS2APP mice compared to its WT controls (Supplemental Table 2). We found 14 BP, $8 \mathrm{CC}$ and $14 \mathrm{MF}$ in the analysis. Fig. 4A shows the network of GO terms enriched, in which we mapped the degree of connectivity for each term in the node sizes and the pairwise gene overlap (Jaccard coefficient) between terms in the edges. We noted that "exocytosis", "exocytic process", "protein localization to cell periphery", "protein localization to plasma membrane" and "voltage-gated cation channel activity" are the most connected terms in the network (Fig. 4B). On the other hand, the most significantly enriched terms are shown in Fig. 4C, which include processes such as "protein localization to cell periphery and plasma membrane”, “interleukin-6 production and regulation”, “interleukin-6 production and regulation”, "exocytosis” and "neuron death”.

For the comparison of the Tau P301S mice versus WT controls, the functional enrichment analysis using DEGs revealed 95 terms (Supplemental Table 2), which include 56 BP, 25 CC and 14 MF enriched terms. The GO network for Tau P301S shows that the most connected terms are related to cytoskeleton dynamics such as "actin filament binding and organization" and "cell leading edge" (Fig. 4D-E). Among the most significantly enriched terms, we found terms such as "learning”, “cognition”, and "small GTPase mediated signal transduction” (Fig. 4F). Results of the functional enrichment analysis for both animal models are presented in Supplemental Table 2.

\section{Discussion}

Here, we evaluated the association of plasma GFAP levels with $A \beta$ and tau pathologies in CU and CI individuals. We demonstrated that GFAP independently associates with $A \beta$, but not with tau pathology. To understand the impact of $A \beta$ and tau pathology separately in GFAP-positive astrocyte phenotypes, we explored the molecular features of 
astrocytes isolated from the hippocampus of two AD mouse models (PS2APP and Tau P301S). Overall, we observed a limited overlap of DEGs between the $A \beta$ and tau mouse models, indicating the unique nature of GFAP-positive astrocytic responses to different aspects of AD pathology. The findings presented in this study allowed the identification of functional categories and biological processes that are a distinctive response to $A \beta$ or tau burden.

Astrocyte biomarkers have been increasingly investigated in $\mathrm{AD}$, and they have already proved to be consistently altered in AD patients (6). However, the production and consequent release of GFAP by astrocytes in response to $\mathrm{AD}$ pathological events seem heterogeneous. Specifically, while GFAP seems to be more associated with A $\beta$ burden $(8,9,21)$ it does not correlate with tau when corrected for $A \beta$ pathology. On the other hand, studies investigating another astrocyte biomarker - the chitinase-3-like protein 1 (YKL-40) demonstrated that YKL-40 seems to be more associated with tau pathology (22-24). These findings highlight the importance of understanding how astrocytes react to specific aspects of AD pathology.

A large portion of the current knowledge regarding the astrocytic responses to $A D$ pathology was obtained from postmortem studies in patients with dementia. At this point, the human brain is usually affected by several pathological components of AD simultaneously, hampering the understanding of the exact role each one is playing in the disease. The use of animal models provides a unique opportunity to explore isolated pathological features of AD and better comprehend its heterogeneous aspects. Our comparison of GFAP-positive astrocytes from animal models of $A \beta$ and tau pathologies evidenced a scarce overlap of genes between these two transgenic lines. At the moment, only a few studies in the literature aimed at stratifying the unique astrocyte signatures triggered by $A \beta$ or tau $(5,12)$. Wu and colleagues specifically focused on the complement $\mathrm{C} 1 \mathrm{q}$ cascade, demonstrating that tau pathology is enough to activate the classical complement pathway (12). In a broader approach, Jiwaji et al. demonstrated a significant overlap of DEGs between astrocytes from A $\beta$ and tau pathology. Our study and the one by Jiwaji and colleagues are highly complementary since present different methodologies. More specifically, studies used different brain regions (hippocampus vs whole cortex and spinal cord) and cell-sorting protocols that isolated distinct astrocyte populations (GFAP-positive astrocytes vs pan astrocytes) (5). Despite the exciting insights provided by these recent studies, the understanding of astrocyte heterogeneity in AD is still in its early days, and further studies exploring region-specificity and disease/pathology stages are still needed. 
bioRxiv preprint doi: https://doi.org/10.1101/2022.02.25.481812; this version posted February 28, 2022. The copyright holder for this preprint (which was not certified by peer review) is the author/funder, who has granted bioRxiv a license to display the preprint in perpetuity. It is made available under aCC-BY-NC-ND 4.0 International license.

Differential expression analysis in the PS2APP GFAP-positive astrocytes revealed that Aqp11 appeared as the most downregulated gene. In humans, AQP11 co-localizes to the endoplasmic reticulum (ER) and mitochondrial-associated membrane (MAM) to facilitate the transport of hydrogen peroxide $(25,26)$. In the ER, AQP11-mediated peroxide transit seems to prevent toxicity during protein folding (26). The downregulation of this peroxiporin in our analysis suggests the presence of ER stress in PS2APP GFAP-positive astrocytes. Additionally, as APP is synthesized in the ER, alterations in MAMs and ER stress are emerging as important pathological mechanisms of $\mathrm{AD}$ and interesting therapeutic targets to modify protein misfolding and synaptic failure (27-29). Furthermore, the upregulation of ubiquitin-related genes (Uba52, Ubc) may indicate compensatory attempts to mitigate the results of ER stress. Ubiquitination is a major post-translational modification responsible for many actions in the cells, including protein degradation, kinases modification and regulation of signaling pathways. The PPI network analysis also evidenced that ER stress is an important alteration in PS2APP hippocampal astrocytes. In specific, the most connected proteins of this network (Ubc and Uba52) were associated with proteostasis. This functional category showed high linkage with others, especially intracellular trafficking, and DNA/RNA processing. Indeed, a recent systematic review of AD neuropathological literature showed proteostasis among the most consistent functional alterations of the disease, however its specificity for $A \beta$ pathology was not demonstrated (30). Furthermore, Tau P301S astrocytes PPI shows more genes associated with DNA/RNA processing function than PS2APP astrocytes. Site-specific phosphorylation regulates tau and, since nuclear tau protects the DNA (31,32), its dysfunction can alter the chromatin landscape in pathological contexts $(33,34)$. The DNA/RNA processing function contained Myc, Yy1, histone deacetylases, among other genes associated with DNA-binding and epigenetic GO terms. Although astrocyte and neuron chromatin dysfunction have been reported (35), it is not clear how tauinduced astrocyte dysfunction and associated chromatin alterations may influence other pathological features of AD.

The differential expression analysis of the Tau P301S astrocytes revealed dysregulation of insulin-related genes and downstream signaling kinases. Indeed, Ins1/2 and Pi3kca, proteins directly involved in regulating energetic brain metabolism, were highly connected and downregulated nodes in the Tau P301S PPI network. In this context, several pathophysiological links have been demonstrated between AD and metabolic disorders such as type 2 diabetes, obesity, and metabolic syndrome in humans and animal models (36-38). Furthermore, it has been demonstrated that when insulin signaling is impaired in astrocytes, 
brain glucose uptake become less efficient, impairing CNS control of glucose homeostasis $(39,40)$. As insulin plays a central role in regulating tau phosphorylation in the brain, deficiencies in insulin signaling could exacerbate neurodegeneration by promoting tau hyperphosphorylation in neurons $(41,42)$. Despite in vitro studies demonstrating that insulin signaling in astrocytes is affected by $A \beta$ exposure $(43,44)$, our findings showed that it does not seem to play a significant role in an animal model presenting $A \beta$ deposition without tau pathology. In fact, the strong link between tau pathology and changes in insulin signaling has been reported in postmortem AD and other human tauopathies (45).

Interpreting transcriptomic changes at the pathway level is more consistent than single gene analysis, and it has the potential to offer insights into the biological processes disturbed in $\mathrm{AD}$. Our functional enrichment analysis identified multiple biological processes driven by each specific aspect of $\mathrm{AD}$ pathology. Exocytosis-related processes are among the most significant biological processes enriched in the PS2APP mice. Interestingly, impaired exocytosis was already reported in $\mathrm{AD}$, specifically in brain regions susceptible to neurodegeneration (46). Corroborating, previous studies have been linking impaired exocytosis in the brain (47), and specifically in astrocytes (48), as a result of $A \beta$ exposure, which might compromise proper neurotransmitter release. Astrocytes play a pivotal role in maintaining synaptic strength via vesicular exocytosis of glutamate and ATP (49). Interestingly, our PPI analysis evidenced neurotransmission as one of the main functional categories disturbed in the PS2APP, but not in the Tau 301S astrocytes. In this sense, abnormalities in astrocyte exocytic activity might represent an underexplored aspect of AD pathology.

Neuroinflammation is now a well-recognized and active process detectable in the early stages of $\mathrm{AD}$. A recent study using the $\left[{ }^{11} \mathrm{C}\right] \mathrm{PRB} 28$ tracer revealed that inflammation and tau propagate jointly in the brain of $\mathrm{AD}$ patients in an $\mathrm{A} \beta$-dependent manner (50). We observed that abnormalities in inflammation-related processes were prominent in both Tau 301S and PS2APP astrocytes. Accordingly, it was previously demonstrated that A $\beta$ and tau are able to independently trigger an inflammatory response that contributes to neurodegeneration in mice $(51,52)$, however, the presence of both pathologies simultaneously potentiates this process (51). Additionally, the release of inflammatory mediators by astrocytes seems to be essential for $A \beta$-induced tau phosphorylation in primary neurons (53). Although microglia are the main players in the brain immune response, astrocytes also respond to CNS threats by releasing a range of cytokines that perpetuate the harmful environment in $\mathrm{AD}(54-56)$. 
The recent advances in understanding the neuropathological features of AD revealed a high degree of complexity in the astrocytic response to this disease. Additionally, the rapid growth in the $\mathrm{AD}$ fluid biomarkers field demonstrated that this complexity is reflected outside the brain. Indeed, comprehending AD blood biomarkers biological interpretation is one of the filed greatest challenges. The paucity of overlap between $A \beta$ - and tau-driven DEGs strikingly shows the specific nature of GFAP-positive astrocyte responses to core AD pathology. Our results revealed multiple astrocyte-related biological processes and potential functional alterations triggered by each aspect of AD pathophysiology. Thus, developing novel biomarkers that reliably reflect $\mathrm{AD}$ heterogeneity seems critical to determine $\mathrm{AD}$ pathology's underpinnings and consequently monitor the efficacy and target engagement of emerging anti-A $\beta$ and anti-tau drugs and develop personalized treatment strategies.

\section{Data sharing}

Datasets used in this study can be accessed via NCBI GEO portal (https://www.ncbi.nlm.nih.gov/geo/). Further intermediate data and codes generated are available from the corresponding author upon request.

\section{Acknowledgements}

We would like to thank the funding agencies that supported this work. ERZ receives financial support from CNPq [460172/2014-0], PRONEX, FAPERGS/CNPq [16/2551-0000475-7], Brazilian National Institute of Science and Technology in Excitotoxicity and Neuroprotection [465671/2014-4], FAPERGS/MS/CNPq/SESRS-PPSUS [30786.434.24734.23112017] and Instituto Serrapilheira [Serra-1912-31365]. BB receives financial support from CAPES [88887.336490/2019-00]. PRN is funded by Fonds de Recherche du Québec - Santé (FRQS; Chercheur Boursier, PR-N and 2020-VICO-279314) and CIHR-CCNA Canadian Consortium of Neurodegeneration in Aging. TKK is funded by the Swedish Research Council (Vetenskåpradet; \#2021-03244), the Alzheimer's Association (\#AARF-21-850325), the BrightFocus Foundation (\#A2020812F), the International Society for Neurochemistry's Career Development Grant, the Swedish Alzheimer Foundation (Alzheimerfonden; \#AF930627), the Swedish Brain Foundation (Hjärnfonden; \#FO2020-0240), the Swedish Dementia Foundation (Demensförbundet), the Swedish Parkinson Foundation (Parkinsonfonden), Gamla Tjänarinnor Foundation, the Aina (Ann) Wallströms and MaryAnn Sjöbloms Foundation, the Agneta Prytz-Folkes \& Gösta Folkes Foundation (\#202000124), the Gun and Bertil Stohnes Foundation, and the Anna Lisa and Brother Björnsson’s 
Foundation. HZ is a Wallenberg Scholar supported by grants from the Swedish Research Council (\#2018-02532), the European Research Council (\#681712), Swedish State Support for Clinical Research (\#ALFGBG-720931), the Alzheimer Drug Discovery Foundation (ADDF), USA (\#201809-2016862), the AD Strategic Fund and the Alzheimer's Association (\#ADSF-21-831376-C, \#ADSF-21-831381-C and \#ADSF-21-831377-C), the Olav Thon Foundation, the Erling-Persson Family Foundation, Stiftelsen för Gamla Tjänarinnor, Hjärnfonden, Sweden (\#FO2019-0228), the European Union’s Horizon 2020 research and innovation programme under the Marie Skłodowska-Curie grant agreement No 860197 (MIRIADE), the European Union Joint Programme - Neurodegenerative Disease Research (JPND2021-00694), and the UK Dementia Research Institute at UCL (UKDRI-1003). TAP is supported by the Alzheimer's Association (grant AACSF-20-648075) and National Institute of Aging (grants R01AG075336, R01AG073267).

\section{Declaration of interest}

HZ has served at scientific advisory boards and/or as a consultant for Abbvie, Alector, Annexon, Artery Therapeutics, AZTherapies, CogRx, Denali, Eisai, Nervgen, Novo Nordisk, Pinteon Therapeutics, Red Abbey Labs, Passage Bio, Roche, Samumed, Siemens Healthineers, Triplet Therapeutics, and Wave, has given lectures in symposia sponsored by Cellectricon, Fujirebio, Alzecure, Biogen, and Roche, and is a co-founder of Brain Biomarker Solutions in Gothenburg AB (BBS), which is a part of the GU Ventures Incubator Program (outside submitted work). The other authors declare that they have no conflict of interest.

\section{Contributors}

MAB: conceptualization, data curation, formal analysis, data interpretation, figures, writing original draft, and writing - review \& editing.

BB: conceptualization, data curation, formal analysis, data interpretation, figures, writing original draft, and writing - review \& editing.

WSB: data curation, formal analysis, data interpretation, figures, writing - review \& editing. DGS: data curation, formal analysis, data interpretation, figures, writing - review \& editing. PCLF: data curation, formal analysis, data interpretation, figures, writing - review \& editing. ASR: data curation, formal analysis, data interpretation, figures, writing - review \& editing. GP: data curation, formal analysis, data interpretation, figures, writing - review \& editing. JPF-S: data interpretation, writing - review \& editing.

ALB: writing - review \& editing. 
NJA: writing - review \& editing.

TKK: writing - review \& editing.

HZ: writing - review \& editing.

KB: writing - review \& editing.

PR-N: writing - review \& editing.

TAP: writing - review \& editing.

ERZ: conceptualization, data interpretation, funding acquisition, supervision, writing original draft, and writing - review \& editing.

\section{References}

1. Siracusa R, Fusco R, Cuzzocrea S. Astrocytes: Role and Functions in Brain Pathologies [Internet]. Vol. 10, Frontiers in Pharmacology . 2019. p. 1114. Available from: https://www.frontiersin.org/article/10.3389/fphar.2019.01114

2. Verkhratsky A, Nedergaard M. Physiology of Astroglia. Physiol Rev. 2018 Jan;98(1):239-389.

3. Escartin C, Galea E, Lakatos A, O’Callaghan JP, Petzold GC, Serrano-Pozo A, et al. Reactive astrocyte nomenclature, definitions, and future directions. Nat Neurosci. 2021 Mar;24(3):312-25.

4. Galea E, Weinstock LD, Larramona-Arcas R, Pybus AF, Giménez-Llort L, Escartin C, et al. Multi-transcriptomic analysis points to early organelle dysfunction in human astrocytes in Alzheimer's disease. medRxiv [Internet]. 2021 Jan $1 ; 2021.02 .25 .21252422$. Available from: http://medrxiv.org/content/early/2021/07/30/2021.02.25.21252422.abstract

5. Jiwaji Z, Tiwari SS, Avilés-Reyes RX, Hooley M, Hampton D, Torvell M, et al. Reactive astrocytes acquire neuroprotective as well as deleterious signatures in response to Tau and Aß pathology. Nat Commun. 2022 Jan;13(1):135.

6. Bellaver B, Ferrari-Souza JP, Uglione da Ros L, Carter SF, Rodriguez-Vieitez E, Nordberg A, et al. Astrocyte Biomarkers in Alzheimer Disease: A Systematic Review and Meta-analysis. Neurology. 2021 May;

7. Carter SF, Herholz K, Rosa-Neto P, Pellerin L, Nordberg A, Zimmer ER. Astrocyte Biomarkers in Alzheimer’s Disease. Trends Mol Med. 2019 Feb;25(2):77-95.

8. Pereira JB, Janelidze S, Smith R, Mattsson-Carlgren N, Palmqvist S, Teunissen CE, et al. Plasma GFAP is an early marker of amyloid- $\beta$ but not tau pathology in Alzheimer's disease. Brain. $2021 \mathrm{Jul}$; 
9. Benedet AL, Milà-Alomà M, Vrillon A, Ashton NJ, Pascoal TA, Lussier F, et al. Differences Between Plasma and Cerebrospinal Fluid Glial Fibrillary Acidic Protein Levels Across the Alzheimer Disease Continuum. JAMA Neurol. 2021 Oct;

10. Landau SM, Lu M, Joshi AD, Pontecorvo M, Mintun MA, Trojanowski JQ, et al. Comparing positron emission tomography imaging and cerebrospinal fluid measurements of $\beta$-amyloid. Ann Neurol. 2013 Dec;74(6):826-36.

11. Maass A, Landau S, Baker SL, Horng A, Lockhart SN, La Joie R, et al. Comparison of multiple tau-PET measures as biomarkers in aging and Alzheimer's disease. Neuroimage. 2017 Aug;157:448-63.

12. $\mathrm{Wu} \mathrm{T}$, Dejanovic B, Gandham VD, Gogineni A, Edmonds R, Schauer S, et al. Complement C3 Is Activated in Human AD Brain and Is Required for Neurodegeneration in Mouse Models of Amyloidosis and Tauopathy. Cell Rep. 2019 Aug;28(8):2111-2123.e6.

13. Patro R, Duggal G, Love MI, Irizarry RA, Kingsford C. Salmon provides fast and biasaware quantification of transcript expression. Nat Methods. 2017 Apr;14(4):417-9.

14. Soneson C, Love MI, Robinson MD. Differential analyses for RNA-seq: transcriptlevel estimates improve gene-level inferences. F1000Research. 2015;4:1521.

15. Walter W, Sánchez-Cabo F, Ricote M. GOplot: an R package for visually combining expression data with functional analysis. Bioinformatics. 2015 Sep;31(17):2912-4.

16. Yu G, Wang L-G, Han Y, He Q-Y. clusterProfiler: an R package for comparing biological themes among gene clusters. OMICS. 2012 May;16(5):284-7.

17. Castro MAA, Wang X, Fletcher MNC, Meyer KB, Markowetz F. RedeR: $\mathrm{R} /$ Bioconductor package for representing modular structures, nested networks and multiple levels of hierarchical associations. Genome Biol. 2012 Apr;13(4):R29.

18. Nelson PT, Alafuzoff I, Bigio EH, Bouras C, Braak H, Cairns NJ, et al. Correlation of Alzheimer disease neuropathologic changes with cognitive status: a review of the literature. J Neuropathol Exp Neurol. 2012 May;71(5):362-81.

19. Szklarczyk D, Gable AL, Lyon D, Junge A, Wyder S, Huerta-Cepas J, et al. STRING v11: protein-protein association networks with increased coverage, supporting functional discovery in genome-wide experimental datasets. Nucleic Acids Res. 2019 Jan;47(D1):D607-13.

20. Gu Z, Gu L, Eils R, Schlesner M, Brors B. circlize Implements and enhances circular visualization in R. Bioinformatics. 2014 Oct;30(19):2811-2.

21. Chatterjee P, Pedrini S, Stoops E, Goozee K, Villemagne VL, Asih PR, et al. Plasma 
glial fibrillary acidic protein is elevated in cognitively normal older adults at risk of Alzheimer’s disease. Transl Psychiatry. 2021 Jan;11(1):27.

22. Alcolea D, Martínez-Lage P, Sánchez-Juan P, Olazarán J, Antúnez C, Izagirre A, et al. Amyloid precursor protein metabolism and inflammation markers in preclinical Alzheimer disease. Neurology. 2015 Aug;85(7):626-33.

23. Antonell A, Mansilla A, Rami L, Lladó A, Iranzo A, Olives J, et al. Cerebrospinal fluid level of YKL-40 protein in preclinical and prodromal Alzheimer’s disease. J Alzheimers Dis. 2014;42(3):901-8.

24. Ferrari-Souza JP, Lukasewicz Ferreira PC, Bellaver B, Tissot C, Wang Y-T, Leffa DT, et al. Astrocyte biomarker signatures of amyloid- $\beta$ and tau pathologies in Alzheimer's disease. medRxiv [Internet]. 2022 Jan 1;2022.01.25.22269841. Available from: http://medrxiv.org/content/early/2022/01/29/2022.01.25.22269841.abstract

25. Takahashi S, Muta K, Sonoda H, Kato A, Abdeen A, Ikeda M. The role of Cysteine 227 in subcellular localization, water permeability, and multimerization of aquaporin11. FEBS Open Bio. 2014;4:315-20.

26. Bestetti S, Galli M, Sorrentino I, Pinton P, Rimessi A, Sitia R, et al. Human aquaporin11 guarantees efficient transport of $\mathrm{H}(2) \mathrm{O}(2)$ across the endoplasmic reticulum membrane. Redox Biol. 2020 Jan;28:101326.

27. Gerakis Y, Hetz C. Emerging roles of ER stress in the etiology and pathogenesis of Alzheimer’s disease. FEBS J. 2018 Mar;285(6):995-1011.

28. Fernandes T, Resende R, Silva DF, Marques AP, Santos AE, Cardoso SM, et al. Structural and Functional Alterations in Mitochondria-Associated Membranes (MAMs) and in Mitochondria Activate Stress Response Mechanisms in an In Vitro Model of Alzheimer’s Disease. Biomedicines. 2021 Jul;9(8).

29. Gao X, Xu Y. Therapeutic Effects of Natural Compounds and Small Molecule Inhibitors Targeting Endoplasmic Reticulum Stress in Alzheimer’s Disease. Front cell Dev Biol. 2021;9:745011.

30. Viejo L, Noori A, Merrill E, Das S, Hyman BT, Serrano-Pozo A. Systematic review of human post-mortem immunohistochemical studies and bioinformatics analyses unveil the complexity of astrocyte reaction in Alzheimer's disease. Neuropathol Appl Neurobiol. 2022 Feb;48(1):e12753.

31. Sultan A, Nesslany F, Violet M, Bégard S, Loyens A, Talahari S, et al. Nuclear tau, a key player in neuronal DNA protection. J Biol Chem. 2011 Feb;286(6):4566-75.

32. Rico T, Gilles M, Chauderlier A, Comptdaer T, Magnez R, Chwastyniak M, et al. Tau 
Stabilizes Chromatin Compaction. Front cell Dev Biol. 2021;9:740550.

33. Gil L, Niño SA, Guerrero C, Jiménez-Capdeville ME. Phospho-Tau and Chromatin Landscapes in Early and Late Alzheimer's Disease. Int J Mol Sci. 2021 Sep;22(19).

34. Nativio R, Donahue G, Berson A, Lan Y, Amlie-Wolf A, Tuzer F, et al. Publisher Correction: Dysregulation of the epigenetic landscape of normal aging in Alzheimer's disease. Vol. 21, Nature neuroscience. United States; 2018. p. 1018.

35. Phipps AJ, Vickers JC, Taberlay PC, Woodhouse A. Neurofilament-labeled pyramidal neurons and astrocytes are deficient in DNA methylation marks in Alzheimer's disease. Neurobiol Aging. 2016 Sep;45:30-42.

36. Li J, Cesari M, Liu F, Dong B, Vellas B. Effects of Diabetes Mellitus on Cognitive Decline in Patients with Alzheimer Disease: A Systematic Review. Can J diabetes. 2017 Feb;41(1):114-9.

37. Sripetchwandee J, Chattipakorn N, Chattipakorn SC. Links Between Obesity-Induced Brain Insulin Resistance, Brain Mitochondrial Dysfunction, and Dementia. Front Endocrinol (Lausanne). 2018;9:496.

38. Diaz A, Escobedo C, Treviño S, Chávez R, Lopez-Lopez G, Moran C, et al. Metabolic Syndrome Exacerbates the Recognition Memory Impairment and OxidativeInflammatory Response in Rats with an Intrahippocampal Injection of Amyloid Beta 1-42. Oxid Med Cell Longev. 2018;2018:1358057.

39. González-García I, Gruber T, García-Cáceres C. Insulin action on astrocytes: From energy homeostasis to behaviour. J Neuroendocrinol. 2021 Apr;33(4):e12953.

40. García-Cáceres C, Quarta C, Varela L, Gao Y, Gruber T, Legutko B, et al. Astrocytic Insulin Signaling Couples Brain Glucose Uptake with Nutrient Availability. Cell. 2016 Aug;166(4):867-80.

41. Gratuze M, Julien J, Petry FR, Morin F, Planel E. Insulin deprivation induces PP2A inhibition and tau hyperphosphorylation in hTau mice, a model of Alzheimer's disease-like tau pathology. Sci Rep [Internet]. 2017;7(1):46359. Available from: https://doi.org/10.1038/srep46359

42. Wood H. Alzheimer disease: Is p-tau the missing link between insulin resistance and AD? Nat Rev Neurol. 2017 Dec;13(12):706.

43. Han X, Yang L, Du H, Sun Q, Wang X, Cong L, et al. Insulin Attenuates BetaAmyloid-Associated Insulin/Akt/EAAT Signaling Perturbations in Human Astrocytes. Cell Mol Neurobiol. 2016 Aug;36(6):851-64.

44. Takano K, Koarashi K, Kawabe K, Itakura M, Nakajima H, Moriyama M, et al. Insulin 
expression in cultured astrocytes and the decrease by amyloid $\beta$. Neurochem Int. 2018 Oct;119:171-7.

45. Rodriguez-Rodriguez P, Sandebring-Matton A, Merino-Serrais P, Parrado-Fernandez C, Rabano A, Winblad B, et al. Tau hyperphosphorylation induces oligomeric insulin accumulation and insulin resistance in neurons. Brain [Internet]. 2017 Dec 1;140(12):3269-85. Available from: https://doi.org/10.1093/brain/awx256

46. $\quad$ Sze CI, Bi H, Kleinschmidt-DeMasters BK, Filley CM, Martin LJ. Selective regional loss of exocytotic presynaptic vesicle proteins in Alzheimer's disease brains. J Neurol Sci. 2000 Apr;175(2):81-90.

47. Yang Y, Kim J, Kim HY, Ryoo N, Lee S, Kim Y, et al. Amyloid- $\beta$ Oligomers May Impair SNARE-Mediated Exocytosis by Direct Binding to Syntaxin 1a. Cell Rep. 2015 Aug;12(8):1244-51.

48. Pham C, Hérault K, Oheim M, Maldera S, Vialou V, Cauli B, et al. Astrocytes respond to a neurotoxic $A \beta$ fragment with state-dependent $\mathrm{Ca} 2+$ alteration and multiphasic transmitter release. Acta Neuropathol Commun [Internet]. 2021;9(1):44. Available from: https://doi.org/10.1186/s40478-021-01146-1

49. Jourdain P, Bergersen LH, Bhaukaurally K, Bezzi P, Santello M, Domercq M, et al. Glutamate exocytosis from astrocytes controls synaptic strength. Nat Neurosci. 2007 Mar;10(3):331-9.

50. Pascoal TA, Benedet AL, Ashton NJ, Kang MS, Therriault J, Chamoun M, et al. Microglial activation and tau propagate jointly across Braak stages. Nat Med [Internet]. 2021;27(9):1592-9. Available from: https://doi.org/10.1038/s41591-02101456-w

51. Pickett EK, Herrmann AG, McQueen J, Abt K, Dando O, Tulloch J, et al. Amyloid Beta and Tau Cooperate to Cause Reversible Behavioral and Transcriptional Deficits in a Model of Alzheimer's Disease. Cell Rep. 2019 Dec;29(11):3592-3604.e5.

52. Laurent C, Dorothée G, Hunot S, Martin E, Monnet Y, Duchamp M, et al. Hippocampal $\mathrm{T}$ cell infiltration promotes neuroinflammation and cognitive decline in a mouse model of tauopathy. Brain. 2017 Jan;140(1):184-200.

53. Garwood CJ, Pooler AM, Atherton J, Hanger DP, Noble W. Astrocytes are important mediators of $A \beta$-induced neurotoxicity and tau phosphorylation in primary culture. Cell Death Dis. 2011 Jun;2(6):e167.

54. Lian H, Yang L, Cole A, Sun L, Chiang AC-A, Fowler SW, et al. NFkB-activated astroglial release of complement C3 compromises neuronal morphology and function 
bioRxiv preprint doi: https://doi.org/10.1101/2022.02.25.481812; this version posted February 28, 2022. The copyright holder for this

associated with Alzheimer’s disease. Neuron. 2015 Jan;85(1):101-15.

55. Zhao J, O’Connor T, Vassar R. The contribution of activated astrocytes to $A \beta$ production: implications for Alzheimer's disease pathogenesis. J Neuroinflammation. 2011 Nov;8:150.

56. Choi SS, Lee HJ, Lim I, Satoh J, Kim SU. Human astrocytes: secretome profiles of cytokines and chemokines. PLoS One. 2014;9(4):e92325. 


\section{Tables}

Table 1. Demographic information

$\begin{array}{ll}\mathrm{CU} & \mathrm{CI} \\ (\mathrm{N}=39) & (\mathrm{N}=51)\end{array}$

\section{Demographics}

\begin{tabular}{lcc}
\hline Age, years, mean (SD) & $71.7(6.07)$ & $71.5(5.92)$ \\
\hline Sex, female, n (\%) & $21(53.8 \%)$ & $19(37.3 \%)$ \\
\hline Education, years, mean (SD) & $16.7(2.34)$ & $16.2(3.04)$ \\
\hline Baseline MMSE score, mean (SD) & $29.4(0.72)$ & $26.3(4.33)$ \\
\hline APOE $\varepsilon 4$ carriers, n (\%) & $15(38.5 \%)$ & $22(43.1 \%)$ \\
\hline
\end{tabular}

Biomarkers

$\left[{ }^{18} \mathrm{~F}\right]$-florbetapir global SUVr, mean (SD) $\quad 1.11(0.158)$

Aß-PET-positive, n (\%) $\quad 14(35.9 \%) \quad 30(58.8 \%)$

$\left[{ }^{18} \mathrm{~F}\right]$-flortaucipir temporal meta-ROI SUVr, mean (SD) $1.19(0.092)$

Tau-PET-positive, n (\%) $\quad 10(25.6 \%) \quad 26(51.0 \%)$

Plasma GFAP, pg/mL, mean (SD) $145(59.2) \quad 154$ (79.5) 


\section{Figure Legends}

Figure 1. Associations of plasma glial fibrillary acidic protein with A $\beta$ and Tau-PET. Association between plasma GFAP and (A) A $\beta$-PET $\left(\left[{ }^{18} \mathrm{~F}\right] \mathrm{AV} 45\right)$ and (C) Tau-PET ( $\left[{ }^{18} \mathrm{~F}\right] A$ V1451). Association of plasma GFAP and with A $\beta$ - and Tau-PET stratified by (B) A $\beta$ and (D) tau positivity $(\mathrm{n}=90)$.

Figure 2. Gene expression analysis in hippocampal astrocytes from AD mouse models. (A) Volcano plots depicting differentially expressed genes (DEGs) in PS2APP (left) or Tau P301S (right) versus WT astrocytes. Insert shows a pie chart of upregulated and downregulated DEG counts. (B) Heatmaps with hierarchical clustering depicting DEGs differentiating PS2APP (left) or Tau P301S (right) versus WT astrocytes. (C) Venn diagram showing DEGs in each model and the matched genes altered in both.

Figure 3. Protein-protein interaction (PPI) networks in PS2APP and Tau P301S astrocytes. PPI network of (A) PS2APP and (C) Tau P301S mapping functional categories of genes observed in enriched Gene Ontology biological processes (GOBP). "NA" denotes genes not observed in any enriched GOBPs. Combined score mapped to edges represents the combined confidence interaction scores provided by the STRING database for each pair of genes (values $>0.7$ are considered high confidence scores). Circos plots showing the connections between functional categories in (B) PS2APP and (D) Tau P301S PPI.

Figure 4. Gene Ontology network for biological processes (BP), cellular component (CC) and molecular function (MF). Gene Ontology (GO) term network of (A) PS2APP and (D) Tau P301S constructed using the proportion of overlapping genes between GO terms (Jaccard Coefficient $>0.15$ ) as edges. Radial plots of 10 top GOBP terms ranked from the network by enrichment significance for (B) PS2APP and (E) Tau P301S. Radial plots of 10 top GO terms ranked from the network by connectivity (degree) for (C) PS2APP and (F) Tau P301S. Inner circle's bar size maps significance of GO term enrichment and color maps the overall direction (upregulation or downregulation) of the term's group of genes.

\section{Supplemental Figure 1. Correlation between GFAP and other AD biomarkers.}

Pairwise correlation matrix between GFAP and seven other known AD biomarkers. Pearson correlation was applied and FDR-adjusted $(\alpha=0.05)$. The strength and direction of the correlation is represented by the color gradient in the upper triangle of the matrix. 
bioRxiv preprint doi: https://doi.org/10.1101/2022.02.25.481812; this version posted February 28, 2022. The copyright holder for this

Coefficients (black) and adjusted p-values (gray) of the pairwise correlations are shown in the lower triangle.

\section{Supplemental Figure 2. Regulation state of PS2APP and Tau P301S PPI astrocyte}

networks. Protein-protein interaction networks mapping regulatory states (up/down regulation) of differentially expressed genes in (A) PS2APP and (B) Tau P301S astrocytes. Combined score mapped to edges represents the combined confidence interaction scores provided by the STRING database for each pair of genes (values $>0.7$ are considered high confidence scores). 
A) GFAP and AB-PET

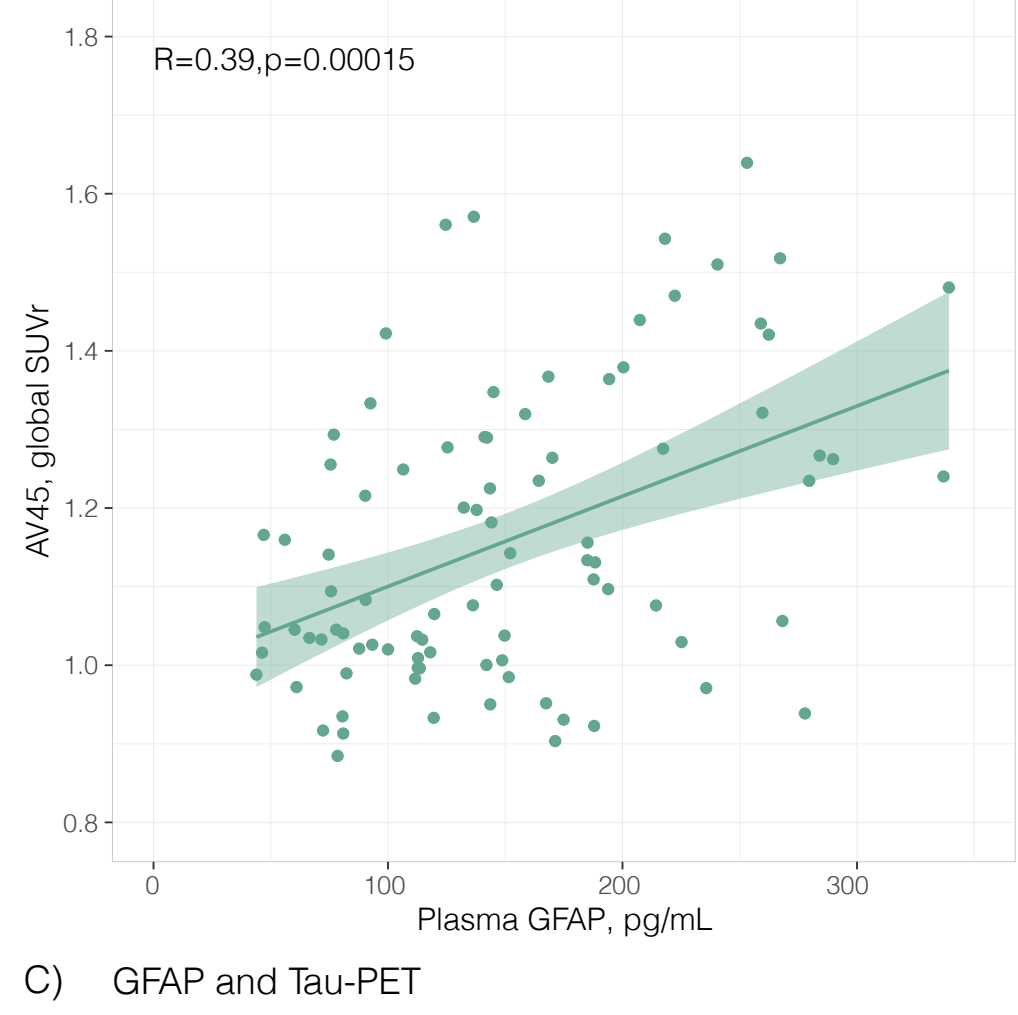

$R=0.35, p=0.00068$

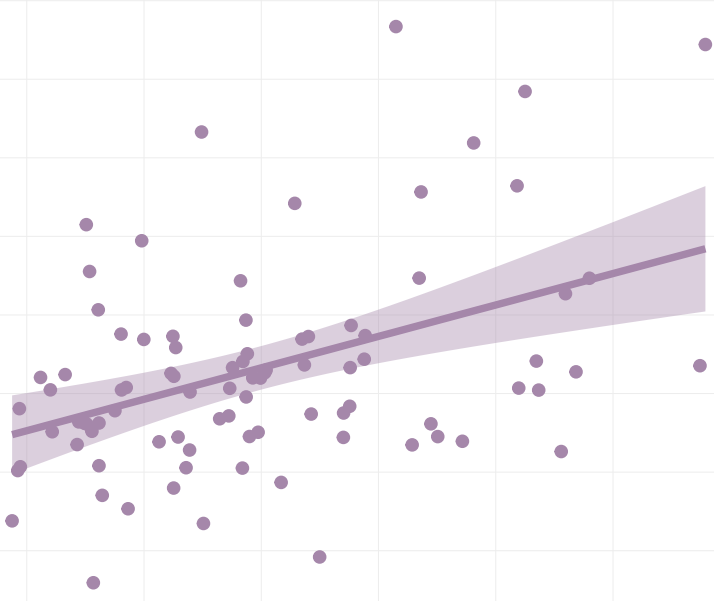

$0.8-$

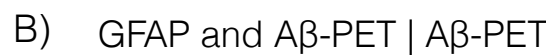

$1.8-\quad \rightarrow A-R=-0.0064, p=0.97$
$\rightarrow A+R=0.34, p=0.024$

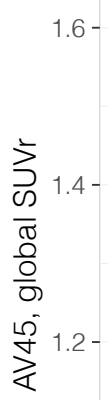

$1.0-$

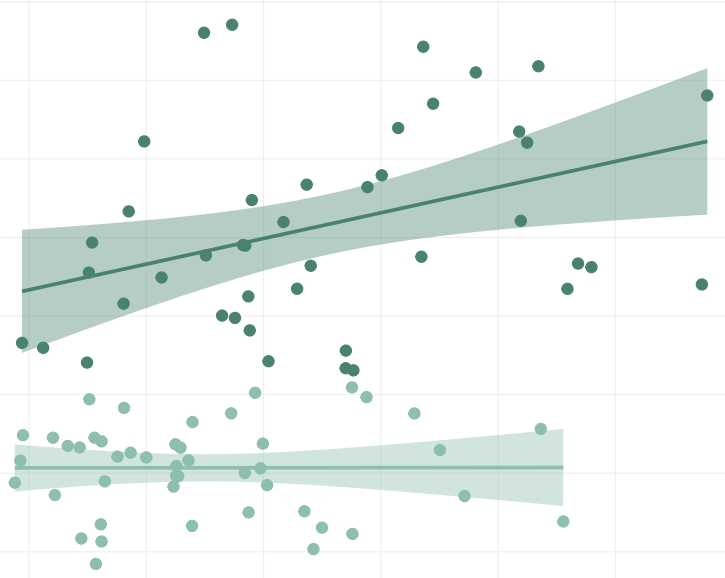

$0.8-$

Plasma GFAP, pg/mL

D) GFAP and Tau-PET | AB-PET

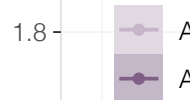

$$
A-R=0.18, p=0.24
$$

$A+R=0.31, p=0.045$

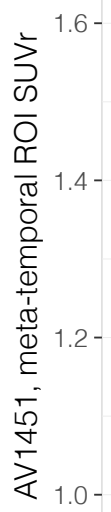

$0.8-$

$$
100
$$


A)
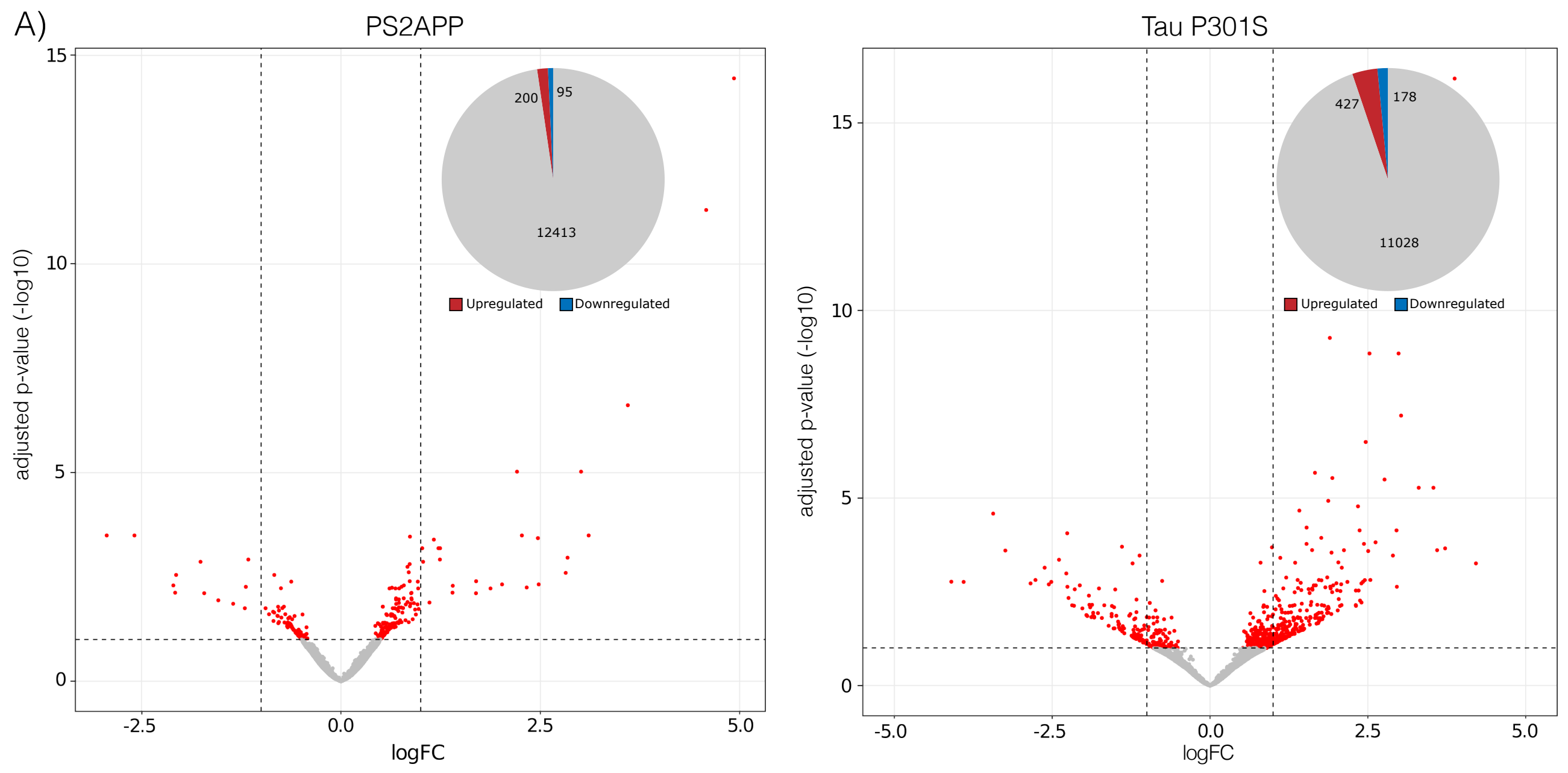

B)

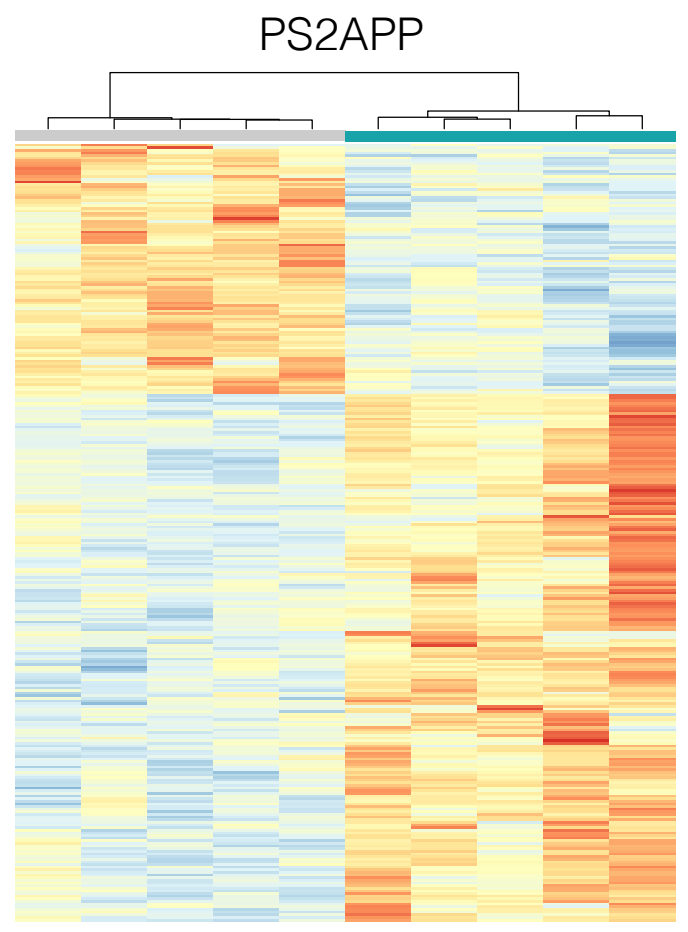

$\square$ Wild Type

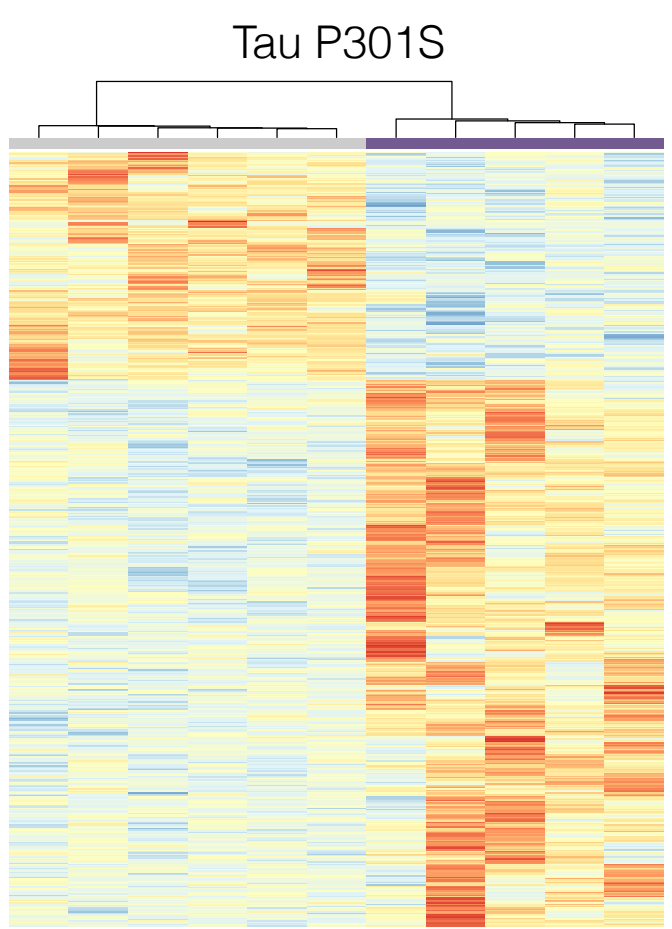

$\square$ Wild Type

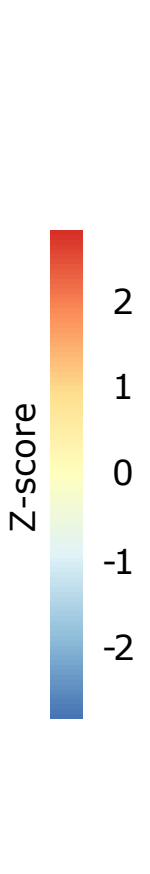

C)

$\square$ Tau P301S

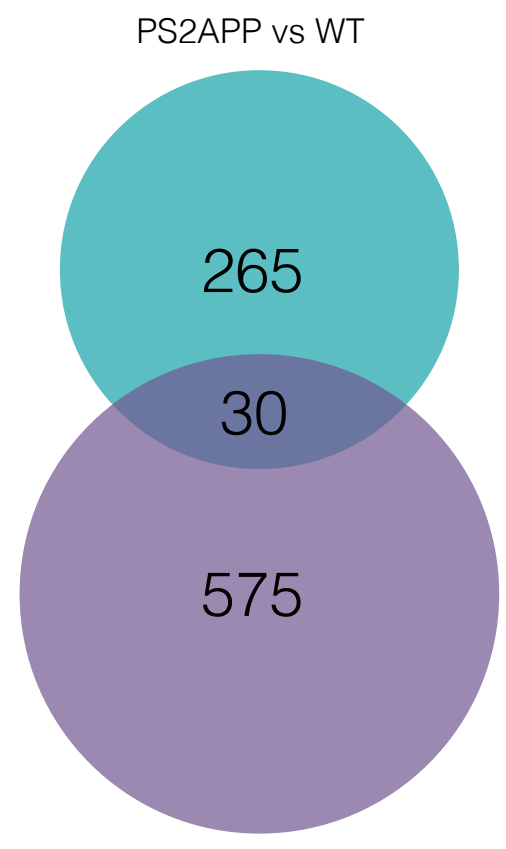

Tau P301S vs WT 
A)

Nckipsd

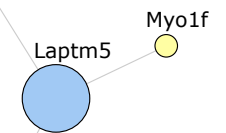

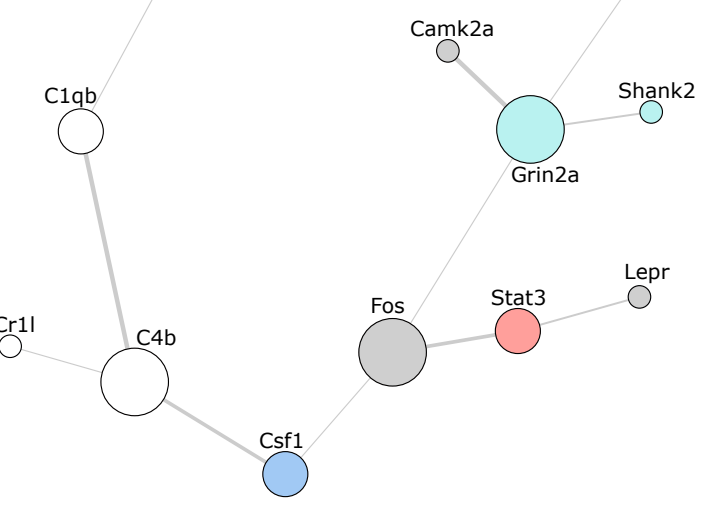

$\stackrel{H 1 F O}{O}$
Combined Score

Node

Degree

25
3
2
1
0

PS2APP

NA
Cytoskeleton
Proteostasis

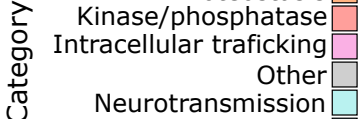

Neurotransmission
Inflammation
DNA/RNA processing $\overbrace{}^{\text {Tubb4a }}$

B)

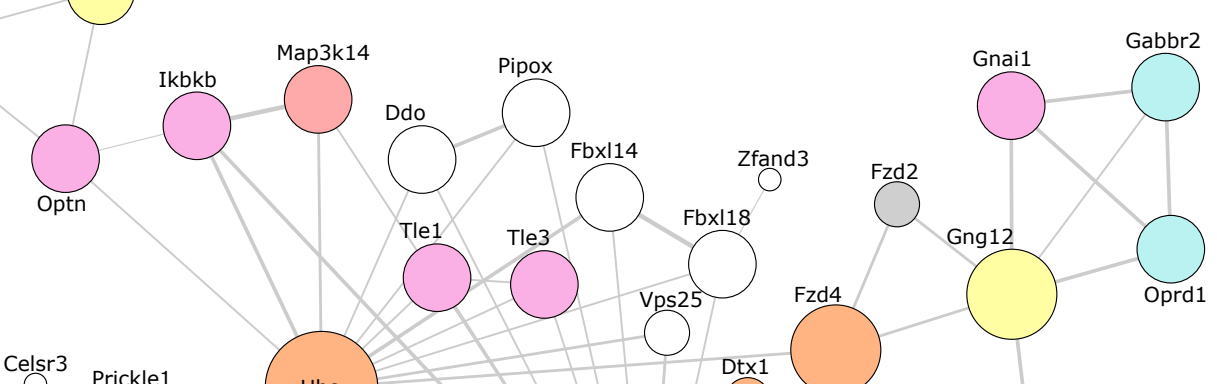

\section{Celsr3 Prickle1}

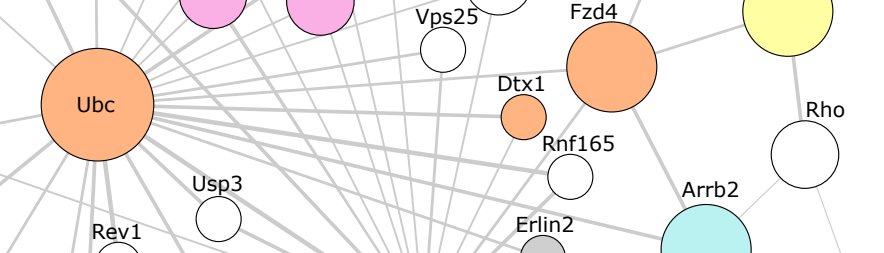

Abli

C)

$$
\text { C) }
$$$$
\bigodot_{\text {Cdk9 }} \bigcirc_{G t+2 a 2}
$$

\section{Tau P301S}

Node

Degree

$2{ }_{2}^{-18}$

$$
\text { (Ex) }
$$

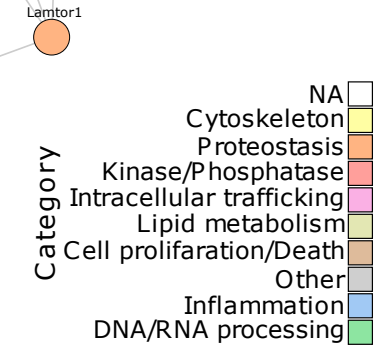

Proteostasis
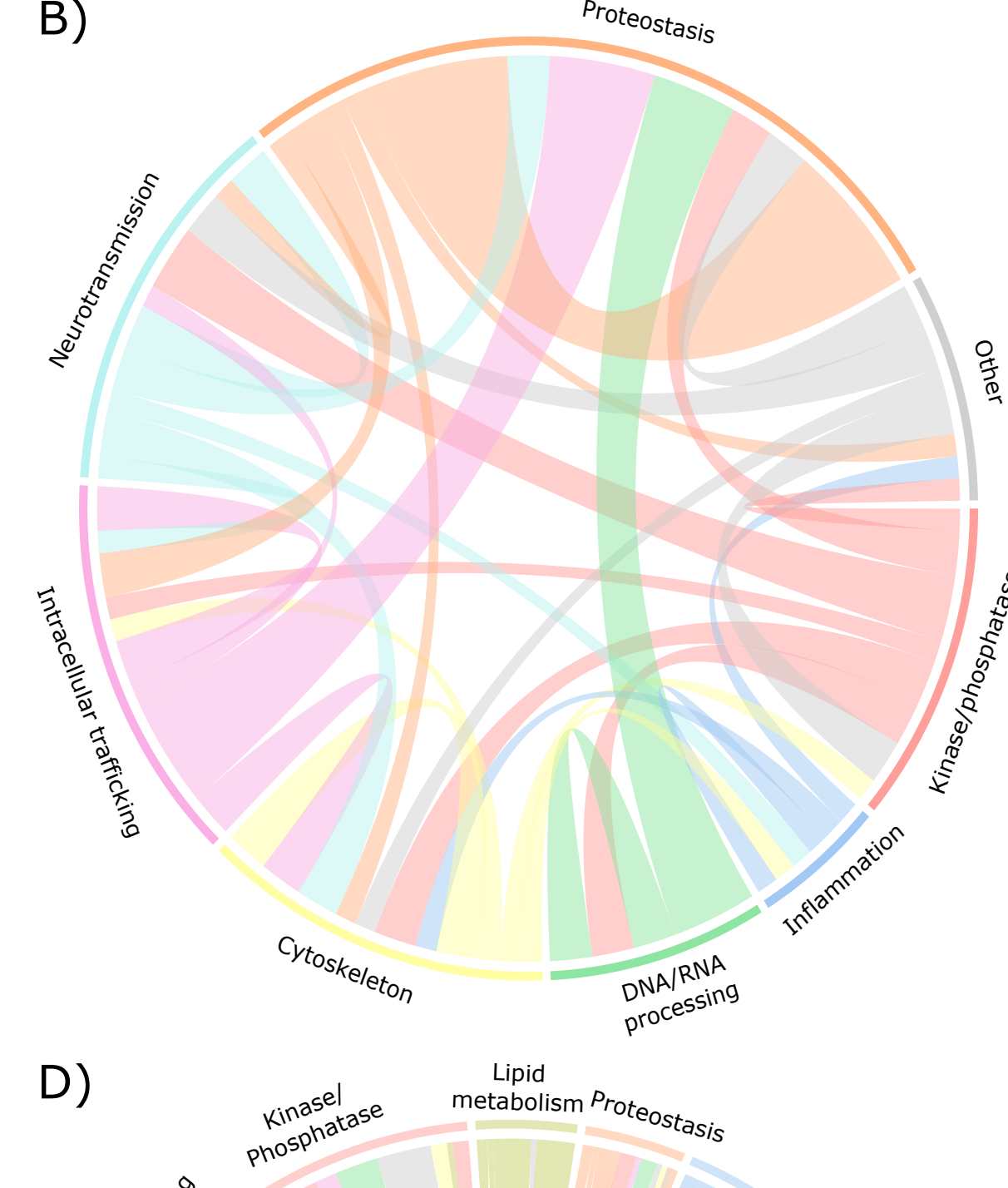

章

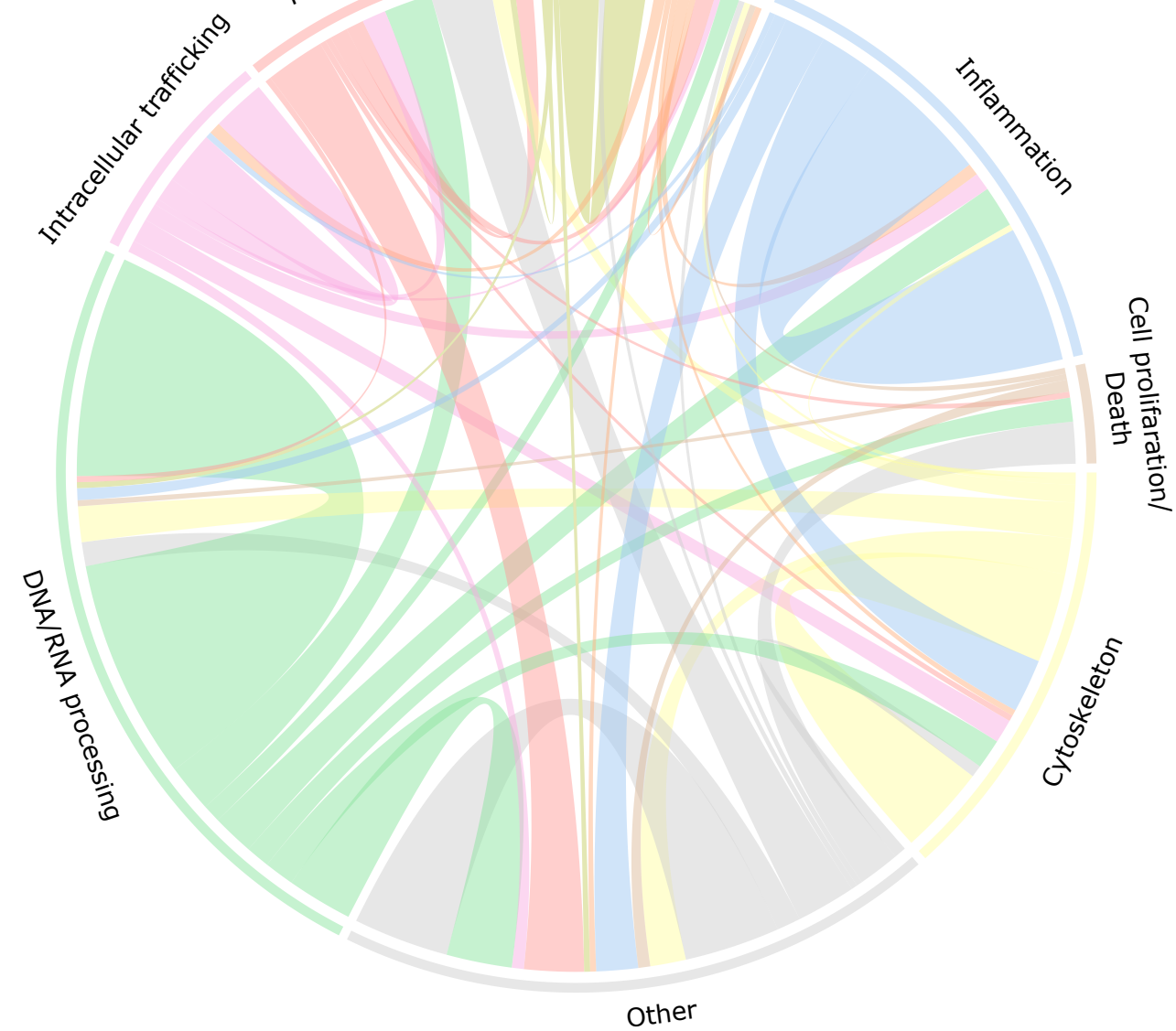




\section{A) \\ PS2APP}

Ubiquitin protein
ligase binding ligase bindi

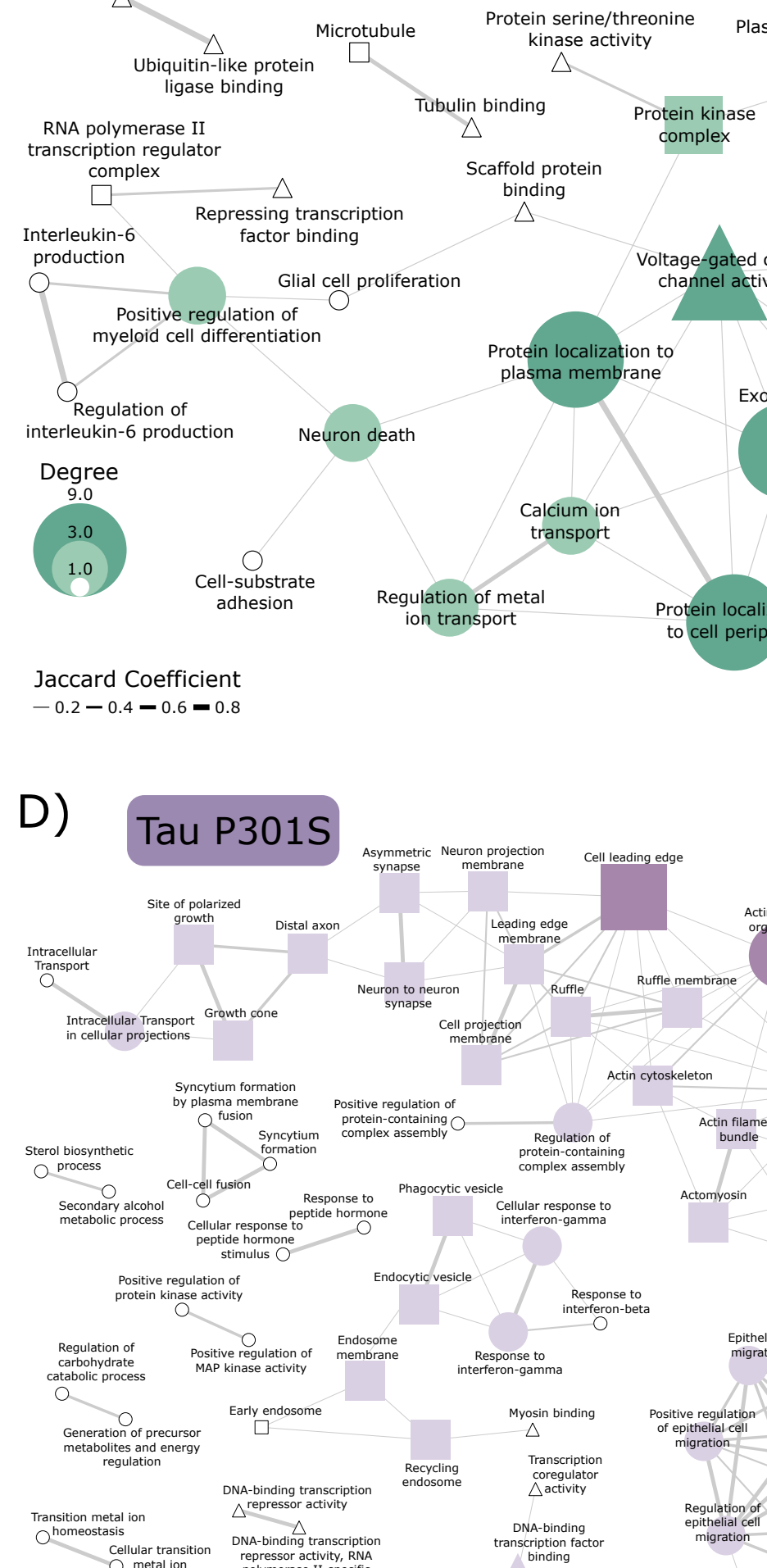

Jaccard Coefficient $-0.2-0.4-0.6-0.8$ Protein localization to
plasma membrane

D)

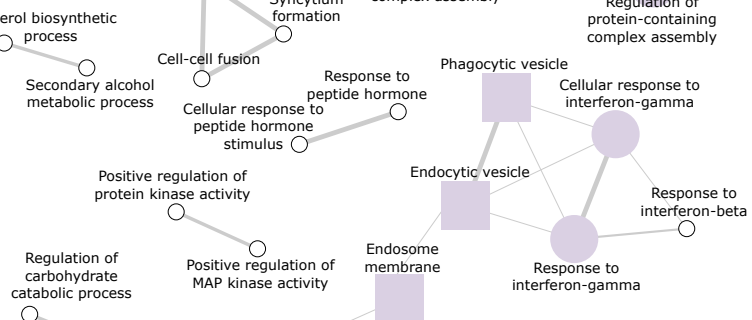
catabo Generation of precursor
metabolites and energy
regulation Early endosome DNA-binding transcription
$\triangle$

Transition metal ion
homeostasis DNA-binding transcription
repressor activity, RNA

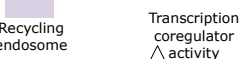

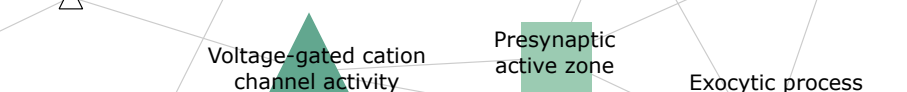
$\begin{array}{ll}\text { Voltage-gated cation } & \text { Presynaptic } \\ \text { active zone }\end{array}$ channel activity Exocytic process
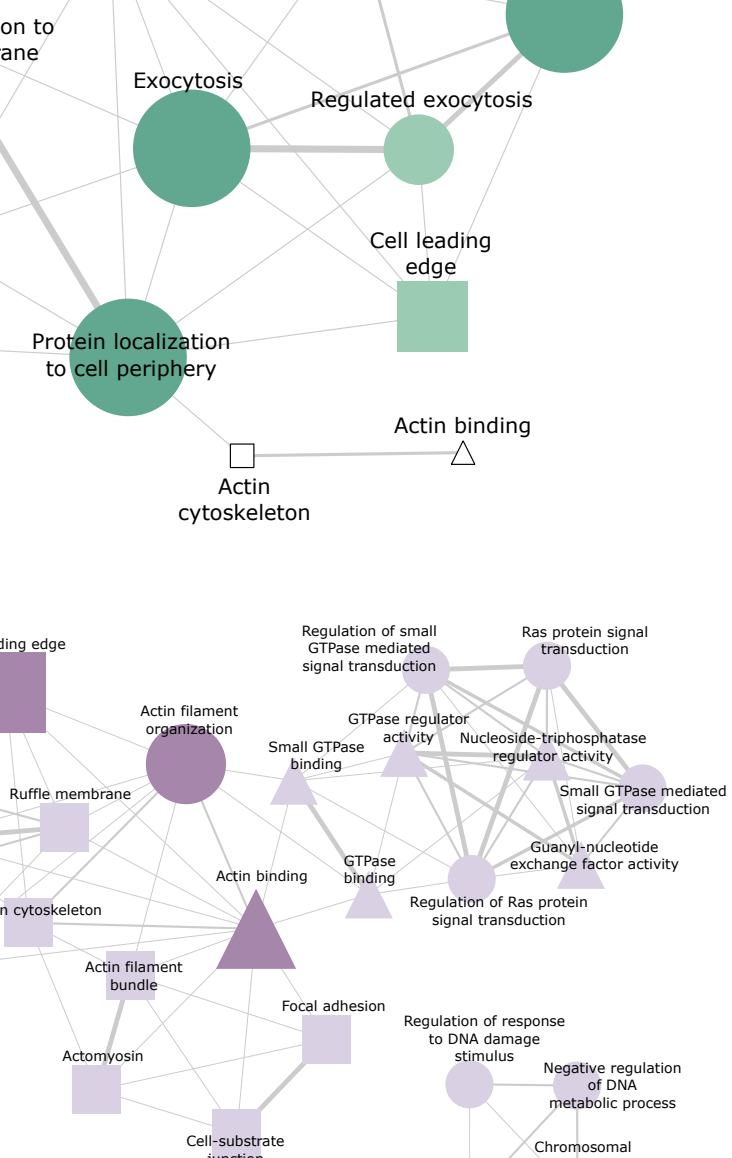

Degree
12.0
4.0
1.0

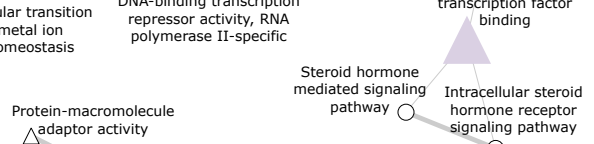
Positive regulation
of cell development Epithelium
migration

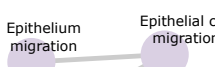
$\underset{\substack{\text { Molecular adaptor } \\ \text { activity }}}{\Delta}$

$$
\begin{gathered}
\text { Multicellular } \\
\text { organism growth }
\end{gathered}
$$

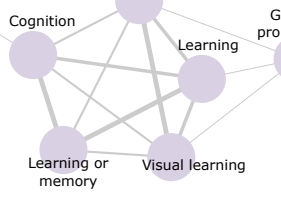

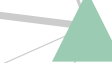
1
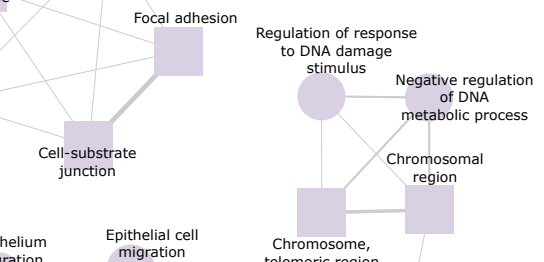

B)

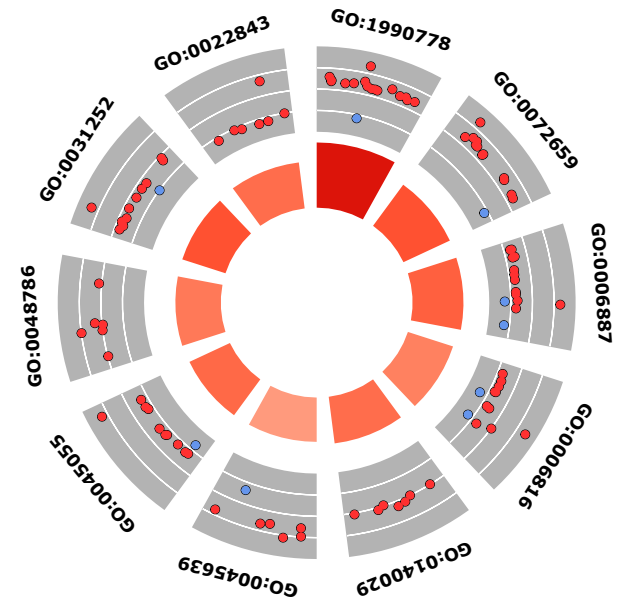

\begin{tabular}{|c|c|}
\hline ID & Description \\
\hline GO:0022843 & voltage-gated cation channel activity \\
\hline GO:0006887 & exocytosis \\
\hline GO:1990778 & protein localization to cell periphery \\
\hline GO:0072659 & protein localization to plasma membrane \\
\hline GO:0140029 & exocytic process \\
\hline GO:0045639 positive regulation of myeloid cell differentiation \\
\hline GO:0045055 & regulated exocytosis \\
\hline GO:0048786 & presynaptic active zone \\
\hline GO:0006816 & calcium ion transport \\
\hline GO:0031252 & cell leading edge \\
\hline
\end{tabular}

E)

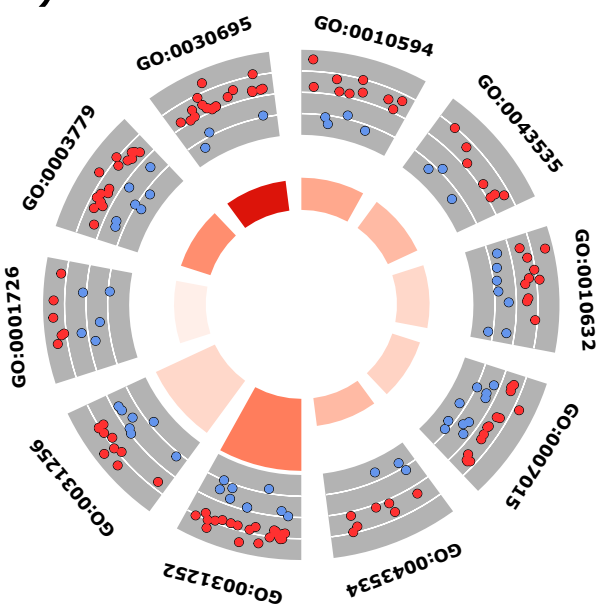

\begin{tabular}{|c|c|}
\hline ID & Description \\
\hline GO:0003779 & actin binding \\
\hline GO:0007015 & actin filament organization \\
\hline GO:0031252 & cell leading edge \\
\hline GO:0043534 & $\begin{array}{c}\text { blood vessel endothelial cell migration } \\
\text { regulation of blood vessel endothelial } \\
\text { cell migration }\end{array}$ \\
\hline GO:0043535 & \begin{tabular}{c} 
ruffle \\
\hline$G O: 0001726$
\end{tabular} \\
\hline GO:0010594 & regulation of endothelial cell migration \\
\hline GO:0010632 & regulation of epithelial cell migration \\
\hline GO:0030695 & GTPase regulator activity \\
\hline GO:0031256 & leading edge membrane \\
\hline
\end{tabular}

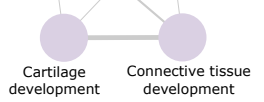

Ontology
C)

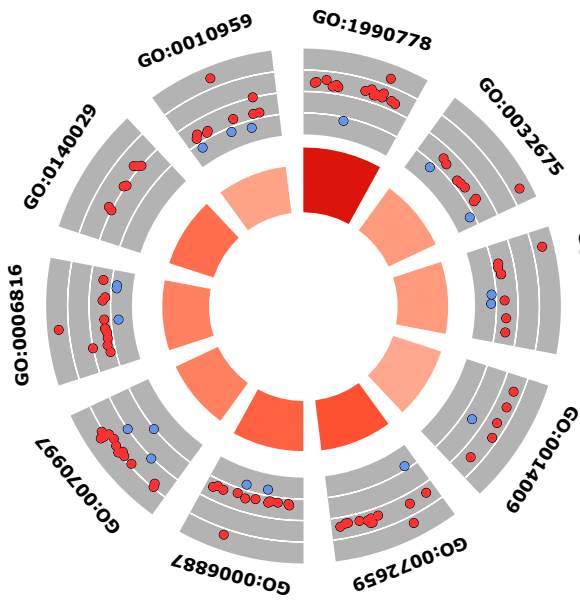

\begin{tabular}{|c|c|}
\hline ID & Description \\
\hline GO:1990778 & protein localization to cell periphery \\
GO:0032675 & regulation of interleukin-6 production \\
GO:0032635 & interleukin-6 production \\
GO:0014009 & glial cell proliferation \\
GO:0072659 & protein localization to plasma membrane \\
GO:0006887 & exocytosis \\
GO:0070997 & neuron death \\
GO:0006816 & calcium ion transport \\
GO:0140029 & exocytic process \\
\hline
\end{tabular}

F)

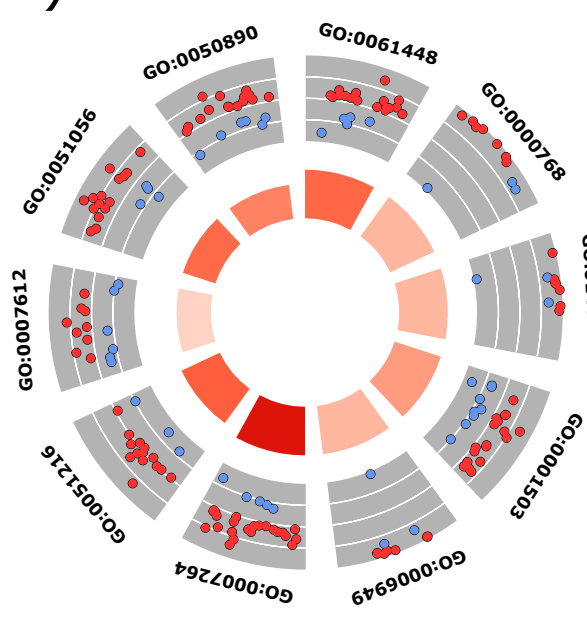

\begin{tabular}{|c|c|}
\hline ID & Description \\
\hline GO:0061448 & $\begin{array}{c}\text { connective tissue development } \\
\text { syncytium formation by plasma } \\
\text { membrane fusion }\end{array}$ \\
\hline GO:0000768 & cell-cell fusion \\
GO:0140253 & ossification \\
GO:0001503 & syncytium formation \\
GO:0006949 & small GTPase mediated signal transduction \\
GO:0007264 & cartilage development \\
GO:0051216 & learning \\
GO:0007612 & regulation of small GTPase mediated \\
GO:0051056 & signal transduction \\
GO:0050890 & cognition \\
\hline
\end{tabular}




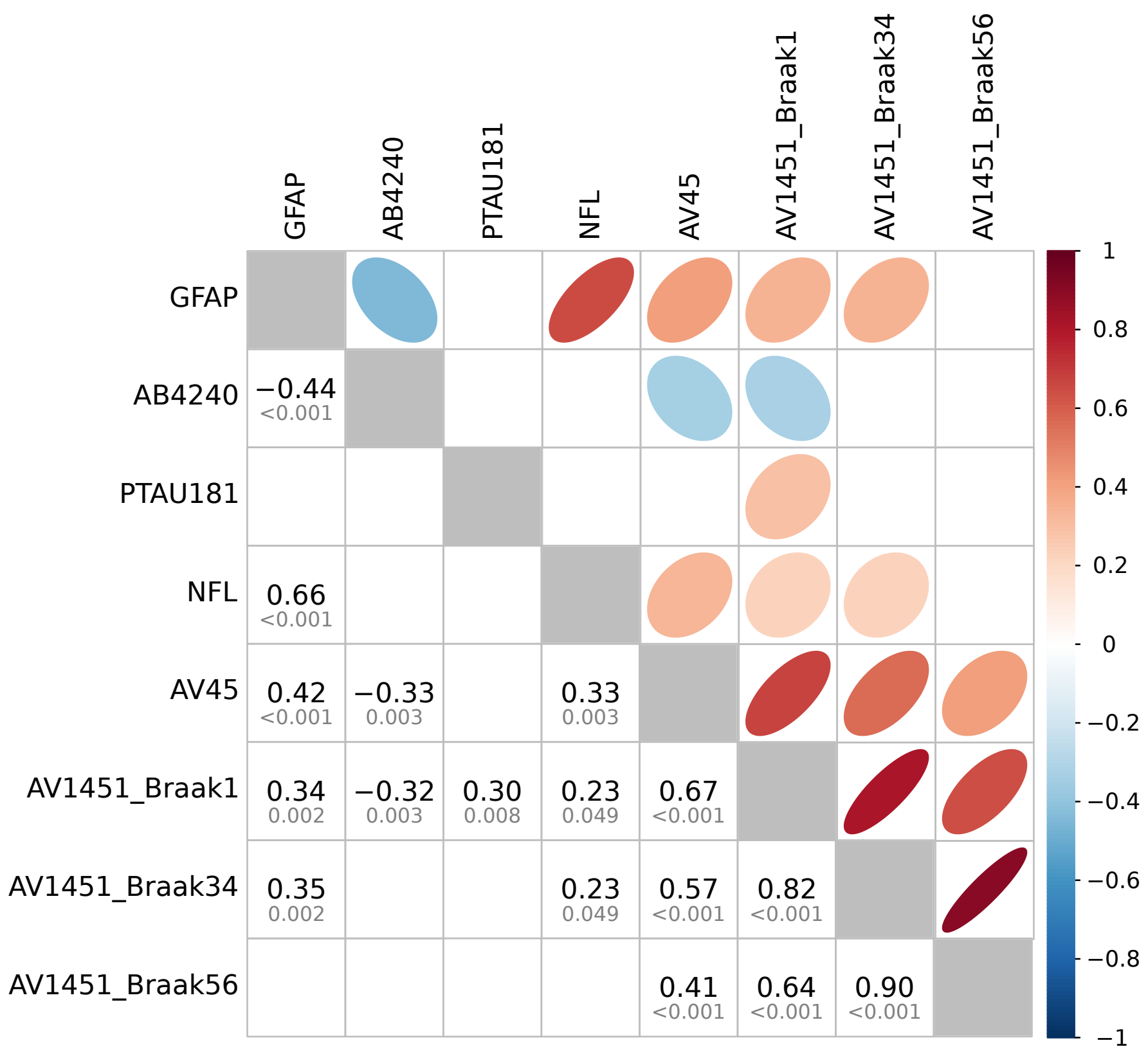


